
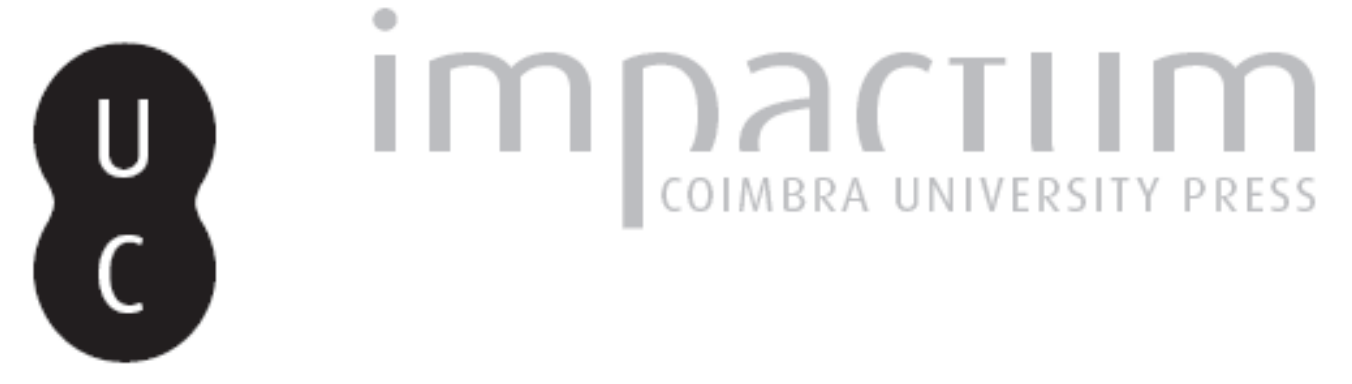

\title{
Exégesis de los monarcas ptolemaicos en Plutarco: interpretación de los personajes en las traducciones europeas
}

\author{
Autor(es): Durán Mañas, Mónica \\ Publicado por: International Plutarch Society \\ URL \\ persistente: \\ URI:http://hdl.handle.net/10316.2/40751 \\ DOI: \\ DOI:http://dx.doi.org/10.14195/0258-655X_4_3
}

Accessed : $\quad$ 26-Apr-2023 14:07:02

A navegação consulta e descarregamento dos títulos inseridos nas Bibliotecas Digitais UC Digitalis, UC Pombalina e UC Impactum, pressupõem a aceitação plena e sem reservas dos Termos e Condições de Uso destas Bibliotecas Digitais, disponíveis em https://digitalis.uc.pt/pt-pt/termos.

Conforme exposto nos referidos Termos e Condições de Uso, o descarregamento de títulos de acesso restrito requer uma licença válida de autorização devendo o utilizador aceder ao(s) documento(s) a partir de um endereço de IP da instituição detentora da supramencionada licença.

Ao utilizador é apenas permitido o descarregamento para uso pessoal, pelo que o emprego do(s) título(s) descarregado(s) para outro fim, designadamente comercial, carece de autorização do respetivo autor ou editor da obra.

Na medida em que todas as obras da UC Digitalis se encontram protegidas pelo Código do Direito de Autor e Direitos Conexos e demais legislação aplicável, toda a cópia, parcial ou total, deste documento, nos casos em que é legalmente admitida, deverá conter ou fazer-se acompanhar por este aviso.

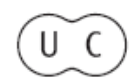


PLOUTARCHOS, n.S. Scholarly Journal of 18

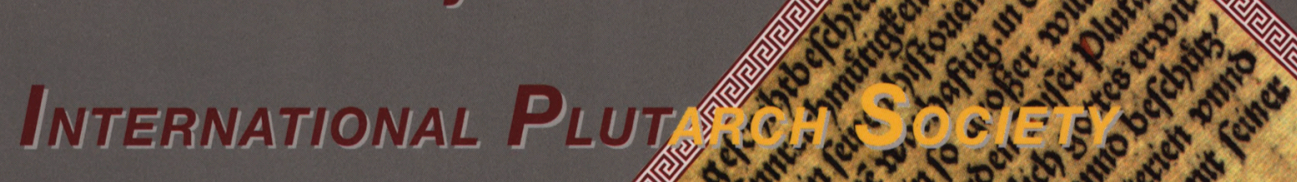

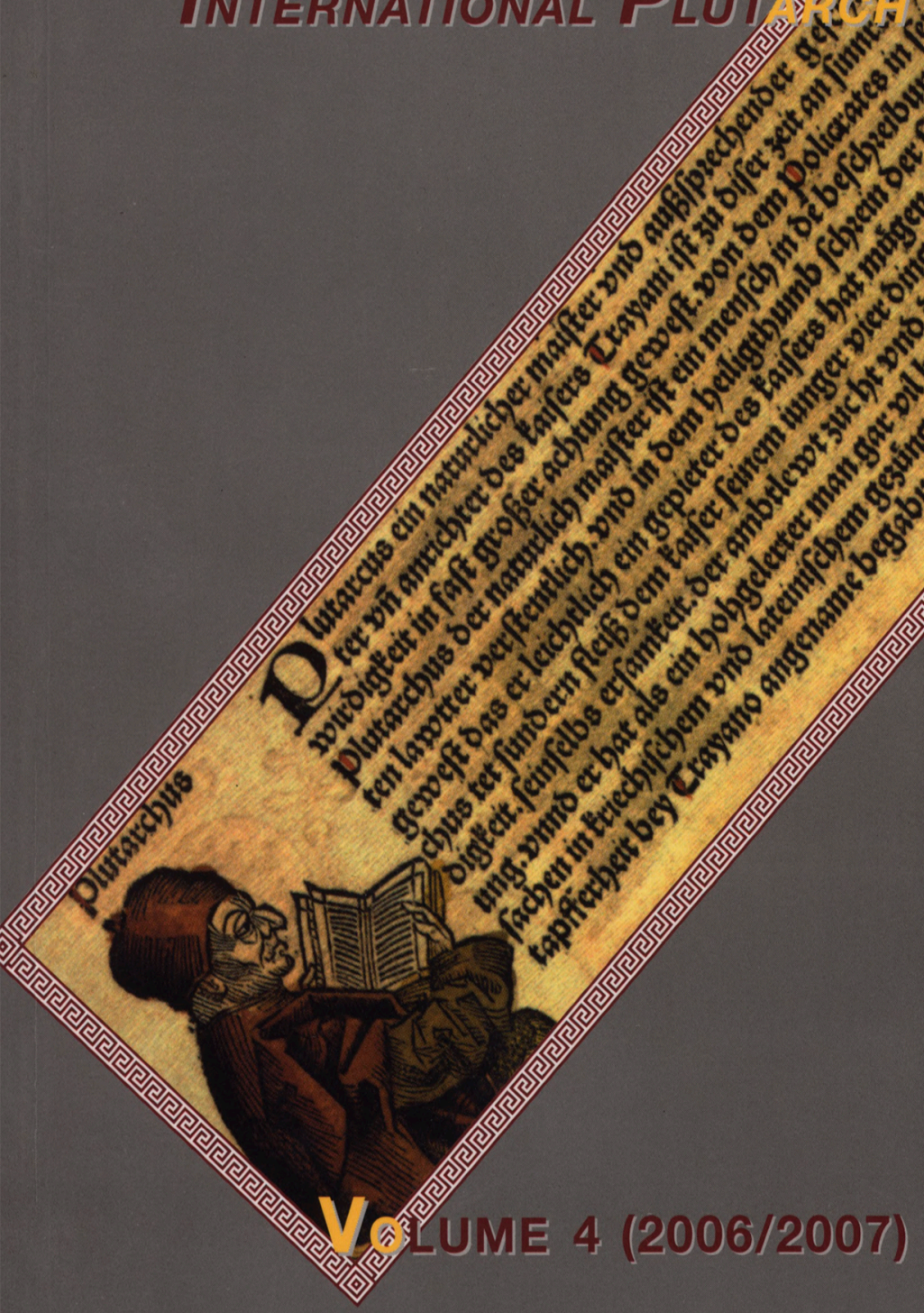

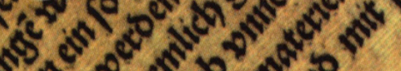

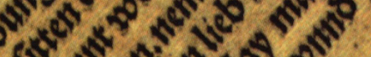

$120^{2} 0^{2} x^{2} x^{2}$

$4 \sin ^{\circ} \cos ^{\circ}$

singes

$0^{2}+0^{2} \mathrm{~s}^{\circ}$

$a^{5} x^{2}$

$s^{5}+\omega^{\circ}$

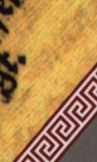

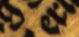

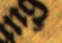




\title{
Exégesis de los monarcas ptolemaicos en Plutarco: Interpretación de los personajes en las traducciones europeas por \\ Mónica Durán Mañas \\ I.E.S. "Padre Sarmiento" \\ Villafranca del Bierzo (León)
}

\begin{abstract}
When Plutarch writes about the Ptolemaic kings he doesn't use to specify their nicknames so it's often difficult to realise whom he is talking about. This paper will try to clear some confusions in relation to this lack of precision in Plutarch's texts and analyse reflections of those interpretation problems in the European translations.
\end{abstract}

La figura de los monarcas ptolemaicos está presente en no pocos lugares de la obra plutarquea, de tal forma que adquiere más relevancia - por el carácter intrínseco de su contenido- en las Vitae que en Moralia. Plutarco nos habla de casi todos ellos y, cuando lo hace, menciona tan sólo su nombre propio sin concretar, en la mayoría de las ocasiones, su apodo ni su numeración: Ptolomeo I Soter, rey de Egipto y fundador de la dinastía (323-283 a.C.) $)^{1}$; Ptolomeo II Filadelfo (283-246 a.C.); Ptolomeo III Evergetes (246-222 a.C.); Ptolomeo IV Filopátor (221-205 a.C.); Ptolomeo V Epífanes (205-181 a.C.); Ptolomeo VIII Evergetes II (146117 a.C.); Ptolomeo IX Látiro (117-81 a.C.); Ptolomeo XII Auletes (80-51 a.C.) y Ptolomeo XIII (51-47 a.C.). Esta circunstancia plantea algunas dificultades para la interpretación de determinados pasajes protagonizados por los monarcas de la dinastía ptolemaica. Partiendo de este hecho, mi objetivo, en las líneas que siguen, se centra en destacar la importancia de una correcta exégesis para la adecuada traducción de un texto. En ningún caso pretendo, con mis observaciones, un acercamiento incisivo a la labor de los traductores europeos, pues considero a éstos más bien víctimas de los comentaristas en quienes se ven obligados, no pocas veces, a delegar la tarea exegética. Así pues, paso a analizar algunos detalles.

Es bien sabido que la monarquía helenística de Egipto toma la costumbre faraónica de llamar a la mayoría de los 
miembros de la dinastía del mismo modo $y$, en consecuencia, surge la necesidad de distinguirlos mediante otros recursos como son los sobrenombres o la clasificación numérica. De esta forma, cada monarca es caracterizado con un apodo que define una hazaña realizada, un rasgo físico característico o una virtud destacable. Así, según Plutarco en Cor. 11.2-3, Ptolomeo I recibió el sobrenombre de Soter por una acción; Ptolomeo II fue llamado Filadelfo por ser amante de su hermana; por su virtud, Ptolomeo III fue denominado Evergetes y, por un rasgo físico, Ptolomeo VIII fue apodado Fiscón, del mismo modo que a algunos reyes las burlas les valieron motes como a Ptolomeo IX Látiro. Paralelamente, los nombres de los reyes helenísticos, así como los de las reinas, comenzaron a hacerse habituales, de forma que influyeron, no sólo en la onomástica de las clases populares, sino también en las más diversas esferas de la vida cotidiana ${ }^{2}$. Por esta razón, encontramos otros personajes que, con el mismo nombre, no guardan ninguna relación con los reyes, como el adivino Ptolomeo, de época algo posterior, en Galb. 23.

En este sentido, los escritos plutarqueos ofrecen ciertas dificultades de interpretación pues, según acabamos de ver, cuando el de Queronea se refiere a un monarca, casi nunca especifica su sobrenombre, de tal forma que debemos deducir por el contexto de cuál de ellos se trata $^{3}$. Pero esto no es tarea fácil, pues, en ocasiones, el propio autor comete errores -especialmente en cuestiones de cronología-. Así, por ejemplo, al hablar de la acogida que dispensó Ptolomeo a Lúculo, en Luc. 2.7, el monarca es descrito como tò $\mu \in \iota$ pákıov

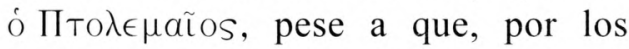
hechos narrados, debe tratarse necesariamente de Ptolomeo IX Látiro quien, en aquel momento -corría el año 86 a.C.-, contaba con 56 años $^{4}$. A veces, esta falta de precisión provoca verdaderos debates entre los estudiosos, como sucede en el caso de un Ptolomeo en Cor. 11.3 a quien tanto Crespo (1999, p. 426 , n. 25) como Flacelière-Chambry (1964, p. 250) identifican con Ptolomeo X Látiro, mientras que Simon (1983, p. 283) lo interpreta como el IX. En cambio, para Perrin (1968, p. 465) se trata de Ptolomeo VIII: "Lathyrus ... Ptolemy VIII, king of Egipt 117-81 B.C., surnamed also Soter and Philometor". No deja de ser significativo el que todos

2 Así, por ejemplo, Plutarco se refiere a unos vasos exhibidos en procesión llamados Antigónidas y Seléucidas por el nombre del monarca que los había hecho fabricar primero (Aem. 32). También en Demetr. 10 los atenienses añadieron dos tribus, la Demetríade y la Antigónide, tras la proclamación de estos personajes como reyes.

3 Sin embargo, cuando habla de algún Ptolomeo que no es un rey de Egipto Plutarco sí suele especificarlo con el fin de no dar lugar a confusiones. Así sucede cuando habla de Ptolomeo, rey de Chipre en Cat.Min., 36.1 o de Ptolomeo, hijo de Crisermo en Cleom. 36.2.

4

Cf. Flacelière-Chambry, 1972, p. 270. 
ellos coincidan en la identificación del monarca por su apodo Látiro, lo cual constituye un criterio unificador, pese a las divergencias en su numeración. En este punto tampoco debe pasarnos inadvertido el hecho de que se establecen dos numeraciones diferentes para los monarcas ptolemaicos a partir de Ptolomeo VI Filométor, dado que hay quienes toman en consideración la primera estancia en el trono de su hermano Ptolomeo VIII Evergetes II $^{5}$. A fin de que esto quede claro, repasemos brevemente el orden cronológico de sucesión en el trono: durante el reinado de Ptolomeo VI Filométor (181-145 a.C.), su hermano Ptolomeo disfrutó de una pequeña estancia en el poder entre los años $170 \mathrm{y}$ 163 a.C. 6 , según lo cual debería ser numerado como Ptolomeo VII. De hecho, por ejemplo, Ateneo, XII 73 afirma, con razón, que este Ptolomeo es el sépti-

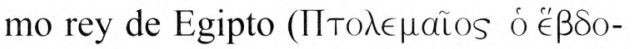

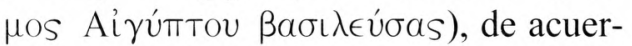
do con la numeración que tiene en cuenta su primer reinado. A continuación, Ptolomeo Neofilopátor, hijo de Ptolomeo VI Filométor, fue asociado al trono por su padre entre los años 162 y 145 a.C., según lo cual debería ser Ptolomeo
VIII. Pero, al morir su padre en el año 145 a.C. y quedar él solo en el poder, fue inmediatamente asesinado por su tío Ptolomeo VII quien volvió a reinar entre el 145 y el 116 a.C. Por tanto, este último rey es, efectivamente el VII y IX rey de Egipto (cf. Numeración cronológica en Fig. 1) pero, por cuestiones prácticas, a menudo se elide su primer reinado a efectos de cómputo y es considerado exclusivamente como el sucesor de su sobrino Ptolomeo VII Neofilopátor (cf. Numeración convencional en Fig. 1).

De acuerdo con esto, en función de la numeración que se tome, se explican las propuestas de Crespo, Flacelière-Chambry y Simon (Ptolomeo X y IX respectivamente), pero en ningún caso la de Perrin (Ptolomeo VIII), aunque siempre cabe la posibilidad de que esta confusión se deba a una simple errata tipográfica y no a un descuido de los intérpretes. Por mi parte, he preferido la numeración convencional para este trabajo, la cual no tiene en cuenta el primer reinado de Ptolomeo VIII, pues considero que no deben numerarse sino los reyes que gobiernan solos en el poder (y, en este sentido, el hijo de Ptolomeo VI es, efectivamente, Ptolomeo VII ya que sí reina

5 Debo precisar que no he hallado ninguna explicación sobre las distintas numeraciones y que, por tanto, esta conclusión no es sino la hipótesis que he podido reconstruir a partir de los datos ofrecidos por los historiadores.

6 Cf. Elvira (1985: 15): “Ante lo desesperado de la situación y la inoperancia del joven monarca (Ptolomeo VI), la plebe alejandrina entregó el poder al hermano menor de Ptolomeo VI, a quien hoy solemos conocer como Ptolomeo VIII, con lo que aún se complicó más la tarea de gobierno. Durante cinco años reinaron a la vez los dos hermanos, junto con la esposa del primero -y hermana de ambos-, Cleopatra II". 


\begin{tabular}{|c|c|c|}
\hline \multicolumn{2}{|c|}{$\begin{array}{l}\text { Numeración cronológica: tiene en cuenta el primer reinado } \\
\text { del hermano de Ptolomeo VI Filométor }\end{array}$} & $\begin{array}{l}\text { Numeración convencional (de referencia en este trabajo): } \\
\text { no tiene en cuenta el primer reinado del hermano de } \\
\text { Ptolomeo VI Filométor. }\end{array}$ \\
\hline Ptolomeo I Soter & (323-283 a.C.) & $(323-283$ a.C. $)$ \\
\hline Ptolomeo II Filadelfo & (283-246 a.C.) & Ptolomeo II Filadelfo \\
\hline Ptolomeo III Evergetes & (246-222 a.C.) & Ptolomeo III Evergetes \\
\hline Ptolomeo IVFilopátor & (221-205a.C.) & Ptolomeo IVFilopátor \\
\hline Ptolomeo VEpífanes & (205-281a.C.) & Ptolomeo VEpífanes \\
\hline Ptolomeo VI Filométor & (181-145 a.C.) & Ptolomeo VI Filométor \\
\hline Ptolomeo VII Evergetes II & (176-163 a.C.) & -- (no se considera su reinado) \\
\hline Ptolomeo VIII Neofilopátor & (162-145 a.C.) & Ptolomeo VIII Neofilopátor \\
\hline (Ptolomeo VII vuelve a reinar & 145-116 a.C.) & Ptolomeo VIII Evergetes II \\
\hline Ptolomeo X Látiro & (116-81 a.C.) & Ptolomeo IX Látiro \\
\hline Ptolomeo XI Alejandro I & (107-88 a.C.) & Ptolomeo X Alejandro I \\
\hline Ptolomeo XII Alejandro II & (80 a.C.) & Ptolomeo XI Alejandro II \\
\hline Ptolomeo XIII Auletes II & (80-51 a.C.) & Ptolomeo XII Auletes II \\
\hline Ptolomeo XIV & (51-47 a.C.) & Ptolomeo XIII \\
\hline Ptolomeo XV & (47-44 a.C.) & Ptolomeo XIV \\
\hline Ptolomeo XVI Cesarión & (44-30 a.C.) & Ptolomeo XV Cesarión \\
\hline
\end{tabular}

Fig. 1: NUMERACIONES DE LOS MONARCAS PTOLEMAICOS 
solo tras la muerte de su padre, si bien por un periodo muy breve).

Algo semejante sucede con dos pasa$\mathrm{jes}^{7}$ en los que un Ptolomeo entrega seis talentos a Arato: Cleom. 19.8 y Arat. 41.5. Por las fechas en que vive Arato (271-213 a.C.), podría tratarse tanto de Ptolomeo II Filadelfo (283-246 a.C.) como de Ptolomeo III Evergetes (246222 a.C), pues aquél vive en los reinados de ambos. Flacelière-Chambry (1976, p. 60, n. 6), Perrin (1975, pp. 453-454) y Manfredini-Orsi (1996, p. 210) proponen el segundo para los dos casos, mientras que, paradójicamente, Simon (1983, p. 283) piensa que Plutarco se refiere a Ptolomeo II Filadelfo en Arat. 41.5 pero a Ptolomeo III Evergetes en Cleom. 19, cuando es evidente que se trata de la misma anécdota. Por su parte, Porter (1979, p. 66, n. 24.4) comenta del Ptolomeo de Arat. 41.5: "he allowed Aratus a pension of six talents per annum, but also about 225 B.C. transferred his patronage to Cleomenes (Polyb. II 51)" $\mathrm{y}$, dada la fecha, inferimos que no puede ser otro más que Ptolomeo III Evergetes. A mi entender, la confusión parte de la falta de precisión cronológica del propio Plutarco en dos pasajes que preceden a los ahora en cuestión. En efecto, en Cleom. 16.2, se afirma que Arato intenta provocar un cambio de planes en los aqueos cuando éste llevaba 33 años como jefe de sus conciudadanos y el mismo número de años aparece cuando, en Arat. 41.2, se dice que hacía 33 años que Arato dirigía los asuntos públicos de los aqueos. Sin embargo, como apuntan Flacelière-Chambry $(1979$, p. 236) respecto a este último pasaje, teniendo en cuenta que la liberación de Sición marca el comienzo de la carrera de Arato en el año 251 a.C. (cf. Pol., II 43.3) y que los acontecimientos narrados ahora se produjeron con certeza en el 224 a.C., no habían pasado más que 27 años, y no 33 . Sea como fuere, tanto en el caso de que hubieran pasado 33 años o 27, el monarca vigente sería, en ambos lugares, Ptolomeo II Filadelfo, rey de Egipto entre el 283 y el 246 a.C. (pues $224+27$ $=251$ y $224+33=257$ ). Por tanto, a partir del comentario de FlacelièreChambry, Simon, en la convicción de que Ptolomeo le daba seis talentos a Arato al comienzo de su carrera, habría calculado las fechas e interpretado que se trataba de Ptolomeo II Filadelfo, quien en 251 a.C. -así como en 257 a.C.- es, efectivamente, rey de Egipto.

En cualquier caso, la hipótesis de Simon no parece descabellada si consideramos otros datos ofrecidos por el propio Plutarco en la misma obra. Por ejemplo, es conocida la ayuda económica que Arato recibió de Ptolomeo II Filadelfo $^{8}$ : en Arat. 12-13, Plutarco

7 Con el fin de obtener una visión general de la situación incluyo, al final, un cuadro recopilatorio (Fig. 2) en el que pueden seguirse con claridad las explicaciones.

8 Cf. Lozano (1989: 25) y Rivet- Drinkwater, s. v. Aratus en OCD, $1996^{3}$. 
refiere cómo Arato había conseguido el favor del rey por enviarle con frecuencia obras de arte, de las que éste gustaba enormemente. Por ello, cuando Arato fue a visitarle, el monarca le regaló 150 talentos, suma que aquél invirtió en mejorar las condiciones de sus ciudadanos (Arat. 13.6). Aunque Plutarco no especifica nada acerca de este Ptolomeo, Flacelière-Chambry (1979, p. 227), en su comentario a Arat., XII 1, afirman que el viaje de Arato a Egipto para solicitar ayuda del monarca se produjo, sin duda, entre los años 251 y 250 a.C., fecha en que gobernaba Ptolomeo II. Además, para las relaciones anteriores de Arato con este Ptolomeo, remiten al pasaje Arat. 4.3, donde, según los mismos autores $(1979$, p. 225$)$ se trata de los monarcas Antígono Gonatas, rey de Macedonia de 277 a 239 a.C. y de Ptolomeo II Filadelfo, rey de Egipto de 283 a 246 a.C. De ello no cabe ninguna duda, pues el asunto se halla bien documentado: cuando Cicerón refiere estos mismos acontecimientos, del texto latino inferimos con plena claridad que se trata de Ptolomeo II Filadelfo "el que reinó el segundo, después de la fundación de la ciudad": cum igitur statuisset opus esse ad eam rem constituendam pecunia Alexandream se proficisci velle dixit remque integram ad reditum suum iussit esse, isque celeriter ad Ptolomaeum, suum hospitem, venit, qui tum regnabat alter post Alexandream conditam (Cic., De Off. 2.82). Respecto a este pasaje, Holden (1966, p. 341), interpreta que Cicerón hace referencia a Ptolomeo
II Filadelfo: "Ptolemy Philadelphus, B.C. 283-247, son of Ptolemy Soter (son of Lagus, B.C. 323-283), who on Alexander's death obtained the government of Egypt"; al tiempo que Miller traduce "and so went in haste to his friend Ptolemy, then upon the throne, the second king after the founding of Alexandria". Y, en nuestra lengua, Guillén (2001, pp. 187-188): "fue rápidamente a visitar a Ptolomeo, que había sido huésped suyo y que en la actualidad era rey, el segundo después de la fundación de Alejandría". Añade este último traductor (2001, p. 188, n.141) una nota explicativa: "Ptolomeo Filadelfo sucedió a su padre Ptolomeo. Reinó en Egipto desde 285 a 247". Consecuentemente, en este punto de la obra plutarquea, no cabe ninguna duda y también otros estudiosos como Simon (1983, p. 283), Perrin (1975: 454), Porter (1979, pp. 93-94) y Manfredini-Orsi (1996, p. 241) coinciden en la misma opinión. En cambio, es destacable la aportación de Walkbank (1979, p. 401), quien, al comentar un pasaje de Polibio (XXIX 24.14) que testimonia las pruebas de amistad entre Arato y Ptolomeo, sin definir al monarca ni por su nombre ni por su numeración, interpreta que el Ptolomeo de Arat. 12-13 es el mismo de Arat. 41.5 a quien todos, a excepción de Simon, habían entendido como Ptolomeo III Evergetes: "Close relations were established at the time of Aratus, who eventually received an annual subsidy of six talents from Alexandria (Plut., Arat. 13.6, 41. 5)". Ahora bien, si el rey de 
Arat. 13 es, en opinión de todos, Ptolomeo II, es razonable pensar que Walkbank también lo interpreta así y que, por tanto, el monarca de Arat. 41. 5 es "el mismo", es decir, Ptolomeo II Filadelfo, lo cual coincide con la hipótesis de Simon y con los cálculos cronológicos anteriores. Pero, si en Arat. 41.5 el autor habla de Filadelfo, consecuentemente, en Cleom. 19.8 se ha de referir al mismo rey, puesto que se trata de la misma anécdota $y$, de este modo, Plutarco estaría refiriéndose en los tres pasajes a Ptolomeo II Filadelfo, por lo que Simon -así como FlacelièreChambry y Perrin- habrían errado al creer que el monarca de Cleom. 19.8 es Ptolomeo III Evergetes. Además, la falta de linealidad en el orden cronológico de la narración contribuye, de forma notoria, a la confusión y a la perplejidad de los comentaristas. Es bien sabido que Plutarco presenta, a menudo, los acontecimientos según conviene a sus intereses, pero en detrimento, en ocasiones, de la fidelidad histórica. Así pues, por ejemplo, cuando en Arat. 24.4 el de Queronea relata que Arato hace de Ptolomeo el aliado de los aqueos dándole la dirección de la guerra por tierra y por mar, hemos de entender que este rey es, necesariamente, Ptolomeo III Evergetes, dado que esta alianza se produce durante su reinado entre los años $240 \mathrm{y}$ 230 a.C. ${ }^{9}$ y así lo entienden también Walkbank (1979, p. 401) -quien no vacila en dar el nombre de Ptolomeo III: "and Ptolemy III had been appointed Hegemon of the Achaean Confederation (Plut., Arat. 24. 4)"-; Perrin (1975, p. 453); Porter (1979, pp. 93-94) y Manfredini-Orsi (1996, p. 241). Pero Simon (1983, p. 283) cree que Plutarco se refiere siempre a Ptolomeo II Filadelfo y, además, una nota a este mismo pasaje Arat. 24.4 en Flacelière-Chambry (1979, p. 97) remite a los capítulos 12-13 como si trataran del mismo monarca, si bien, según hemos visto, se refieren necesariamente a Ptolomeo II, como los propios autores afirman (1979, p. 225). Y, para colmo, esta misma nota a Arat. 24. 4 remite también al capítulo 41.5 referido, según ellos mismos (1976, p. 60, n. 6) a Ptolomeo III Evergetes. De este modo, vemos a los comentaristas contradecirse en sus propias afirmaciones.

Creo detectar un nuevo error en otra interpretación de Simon (1983, p. 284, s.v. Ptolémée XIV), quien identifica con Ptolomeo XIV el dinasta de los pasajes Caes. 49.3 y 9, referidos a la reconciliación de Cleopatra con su hermano Ptolomeo por instigación de César y la posterior desaparición de aquél en la batalla librada junto al Nilo los últimos días de marzo del año 47 a.C. Sin embargo, lo más verosímil es que se trate de Ptolomeo XIII, quien reinó juntamente con su hermana y esposa Cleopatra VII, hasta que ciertas diferencias les llevaron a enfrentarse. Tras ello, César le obligó a compartir de nuevo el

9 Cf. R. M. Errington, s. v. Achaean Confederacy, en $O C D, 1996^{3}$, y Grimal, 1983, p. 147. 
poder con ella y se instaló en su palacio, ante lo cual Ptolomeo se declaró en guerra abierta ${ }^{10}$. Crespo $(1999$, p. 230, n. 129) lo identifica como Ptolomeo XIII, pero Perrin y Flacelière-Chambry se abstienen de opinión en este lugar. Con todo, estos últimos autores (1973, p. 261, n. 1), a propósito del mismo asunto en Pomp. 80.8, atribuyen la desaparición a Ptolomeo XIV, mientras Perrin se mantiene en silencio. Por su parte, Simon (1983, p. 284) interpreta como Ptolomeo XIV también el monarca de este pasaje. Hemos de precisar que los dos hijos de Ptolomeo XII Auletes, esto es, Ptolomeo XIII y Ptolomeo XIV, se casaron con su hermana Cleopatra VII. César se enamoró de ella y la reconcilió con su hermano Ptolomeo XIII para que gobernase juntamente con él (Caes. 49.3) y poder controlar la política de Egipto desde fuera. Aquí introduce Plutarco la derrota de Ptolomeo XIII en el Nilo y su desaparición (Caes. 49.9 y Pomp. 80.8). Después de esta victoria, César casó a Cleopatra con su hermano menor de trece años, Ptolomeo XIV, cuando ella contaba veinte, y les entregó el trono de Egipto, aunque él controlaba el poder, pues era, además, amante de Cleopatra. Fruto de esta unión prohibida nació Cesarión, y Ptolomeo XIV fue asesinado por orden de su hermana en el 44 a.C., tras conocer ésta la muerte de Cé- sar. Según la doble numeración que hemos visto a partir de Ptolomeo VI, Ptolomeo XIII puede ser interpretado, efectivamente, también como Ptolomeo XIV, pero, si Simon se hubiese inclinado por este otro criterio numérico, debería haber considerado a Ptolomeo VIII Evergetes II como Ptolomeo VII, a Ptolomeo IX Látiro como el X, a Ptolomeo X como XI, al XII como XIII, y así sucesivamente, lo cual no sucede en ningún caso ${ }^{11}$. Por su parte, Crespo, que aquí sí coincide con la numeración convencional, en Cor. 11.3 sigue la cronológica.

Una pequeña dificultad más se nos plantea en De fort. rom. 319D. Según este pasaje, César se muestra tan convencido de que la fortuna le acompaña en todo que piensa poder provocar la huida de Pompeyo y su asesinato por parte de Ptolomeo sin mancharse él mismo de sangre. Según el índice de nombres propios en López Salvá (1989, p. 327, s.v. Ptolomeo) -tomado, a su vez, del índice onomástico de la edición de F. C. Babbitt (1962, p. 341)- se trata de Ptolomeo XII, aunque no se determina su apodo ni aparece en la traducción ciertamente ninguna nota que explique este Ptolomeo. FrazierFroidefond (1990, p. 267) optan por interpretar Ptolomeo XIV, si bien otros, como Perrin, se abstienen de juicio al respecto. Por mi parte, creo que se trata más bien de Ptolomeo XIII, rey de Egipto entre los

10 Para una visión general de los acontecimientos de este momento histórico, vid. Der kleine Pauly, IV, s.v. Ptolomeus, $\mathrm{n}^{\circ} .17$.

11 Cf. Simon, 1983, p. 283, s.v. Ptolémée VIII, IX, X, etc). 
años 51 y 47 a.C., puesto que es a éste a quien pide asilo Pompeyo tras la derrota de Farsalia en el 48 a.C. Un dato más ante quem nos ayuda a determinar el momento histórico: Pompeyo había escrito un discurso en griego para Ptolomeo (Pomp. 79.2) pero muere en el año 48 a.C., por lo que difícilmente podría tratarse de Ptolomeo XIV, quien no accede al trono hasta el 47 a.C. Se trataría, por tanto, de Ptolomeo XIII. Vemos, así, que todas estas referencias deben ser sometidas a rigurosa revisión.

\section{Conclusión}

He intentado, en las líneas precedentes, ofrecer una visión panorámica de las dificultades hermenéuticas de algunos pasajes plutarqueos, así como de las soluciones adoptadas por estudiosos principalmente de Francia, Inglaterra y España, recurriendo en ocasiones, a aportaciones procedentes de otras nacionalidades como Italia o Estados Unidos. He observado que las menciones a los monarcas ptolemaicos -más frecuentes en las Vitae- aparecen a menudo sólo con el nombre propio, sin más datos que el contexto para dilucidar la identidad del monarca en cuestión. De este forma, a partir de ciertas divergencias en las numeraciones de los reyes establecidas por los comentaristas, he reconstruido una hipótesis para explicarlo (cf. Fig. 1). A mi modo de ver, es preferible la numeración convencional -que obvia el primer reinado de Ptolomeo VIII asociado a su hermano-, pues aquellos reyes que gobiernan asociados al trono, lo mismo que las reinas, no deben numerarse como tales. Con el fin de resumir el resto de las explicaciones, he recopilado la información en un cuadro que adjunto (Fig. 2), en el que aparecen, en negrita, las interpretaciones divergentes $y$, subrayadas, las coincidencias entre autores cuyas hipótesis son diferentes de las de los demás. Confrontando los dos cuadros (Figs. 1 y 2) entiendo que Flacelière-Chambry utilizan la numeración cronológica -al menos así lo parece en todos los fragmentos estudiados-, mientras que Simon y Crespo fluctúan entre ambas. Para otras interpretaciones que no coinciden con ninguna de las dos numeraciones no tengo respuesta como es el caso de las de Salvá y Perrin, a menos que entendamos un error tipográfico, al menos en el primer caso. Véase el cuadro recopilatorio en la página siguiente.

Por otra parte, es evidente que si Plutarco no especifica a qué Ptolomeo se refiere en cada momento de la narración, este hecho no afecta estrictamente a la ákpíßeta de la traducción -pues los intérpretes se limitan a verter el contenido del texto en otra lengua- pero sí a la ákpíßєıа hermenéutica. El debate comienza, pues, a la hora de dar una interpretación histórica a los textos, para lo cual hemos de contar con los pequeños errores del propio Plutarco, con la falta de un criterio unitario entre los historiadores a la hora de clasificar a los miembros de la dinastía ptolemaica y, por último, con las imprecisiones de los filólogos quienes, no pocas veces, adoptan 


\begin{tabular}{|c|c|c|c|c|c|c|}
\hline $\begin{array}{l}\text { Texto: } \\
\text { Vitae }\end{array}$ & CRESPO & $\begin{array}{l}\text { FLACELIÈRE- } \\
\text { CHAMBRY }\end{array}$ & SIMON & PERRIN $^{12}$ & WALBANK & PORTER \\
\hline Cor. 11.3 & Ptol. X & Ptol. X & Ptol. IX & Ptol. VIII & --- & --- \\
\hline $\begin{array}{c}\text { Cleom. } \\
19.8\end{array}$ & --- & Ptol. III & Ptol. III & $\begin{array}{c}\text { Ptol. } \\
\text { Evergetes }\end{array}$ & --- & --- \\
\hline $\begin{array}{l}\text { Arat. } \\
41.5\end{array}$ & --- & Ptol. III & Ptol. II & $\begin{array}{c}\text { Ptol. } \\
\text { Evergetes }\end{array}$ & El mismo & $\begin{array}{l}\text { Indica en una nota que } \\
\text { es Ptol. Evergetes }\end{array}$ \\
\hline $\begin{array}{l}\text { Arat. } \\
12-13\end{array}$ & --- & Ptol. II & Ptol. II & $\begin{array}{c}\text { Ptol. } \\
\text { Filadelfo }\end{array}$ & $\frac{\text { El mismo }}{(\text { (iPtol.II?)) }}$ & Ptol. Filadelfo \\
\hline $\begin{array}{l}\text { Arat. } \\
24.4\end{array}$ & --- & $\begin{array}{l}\text { Remite a } \\
\text { Arat. 12- } \\
\text { 13: Ptol. II } \\
\text { Remite a } \\
\text { Arat. 41.5: } \\
\text { Ptol. III }\end{array}$ & Ptol. II & $\begin{array}{c}\text { Ptol. } \\
\text { Evergetes }\end{array}$ & Ptol. III & Ptol. Evergetes \\
\hline
\end{tabular}

\begin{tabular}{|c|c|c|c|c|c|c|}
\hline $\begin{array}{c}\text { Caes. } \\
49.3 \text { y } 9\end{array}$ & Ptol. XIII & $\begin{array}{c}\text { Se abstie- } \\
\text { nen }\end{array}$ & Ptol. IVX & Se abstiene & --- & -- \\
\hline $\begin{array}{c}\text { Pomp. } \\
80.8\end{array}$ & --- & Ptol. XIV & Ptol. IVX & Se abstiene & --- & -- \\
\hline
\end{tabular}

\begin{tabular}{|c|c|c|l|}
\hline $\begin{array}{c}\text { Texto: } \\
\text { Moralia }\end{array}$ & SALVÁ & $\begin{array}{c}\text { FrAZIER- } \\
\text { FrOIDEFOND }\end{array}$ & PERRIN \\
\hline $\begin{array}{c}\text { De fort. } \\
\text { Rom. 319D }\end{array}$ & Ptol. XII & Ptol. XIV & Se abstiene \\
\hline
\end{tabular}

Fig. 2: PASAJES CON INTERPRETACIONES DIVERGENTES

una solución de compromiso e incluso para transmitirlo, pues de nada sirve prefieren obviar ciertas cuestiones que poder leer un texto si no se entiende. Por suscitan confusión. Por tanto, aunque la ello, el traductor ha de revestirse de inslabor del traductor resulte filológica- trumentos exegéticos precisos y poseer mente correcta sin adentrarse en los un bagaje de conocimientos que le perlaberintos de la exégesis, considero que mitan un dominio de la materia con la el trabajo del filólogo no puede prescin- que trabaja a fin de alcanzar el mayor dir de una comprensión profunda del grado de precisión posible en su tarea. autor original y ha de zambullirse nece- La traducción se convierte, así, en un sariamente en el contenido de la obra arte y el traductor se alza como $\delta \eta \mu$ เoup- 


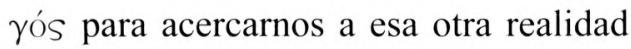
que constituye la obra original.

\section{BIBLIOGRAFÍA}

Aguilar, R. Ma'-Pérez Vilatela, L.,

- Vidas de Sertorio y Pompeyo, Madrid, 2004.

BAввIтT, F. C.,

- Plutarch's Moralia, IV, Loeb, LondresCambridge Mass., 1962

BEVAN, E. R.,

- The house of Ptolemy. A history of Egypt under the ptolemaic dynasty, Chicago, 1968.

BiANCHI BANDINELli, R. (coord.),

- Historia y civilización de los griegos, VII, Barcelona, 1980.

Crespo, E.,

- Plutarco. Vidas paralelas. Alejandro-César. Pericles-Fabio Máximo. AlcibíadesCoriolano, Madrid, 1999.

ELvirA, M. A.,

- El Egipto Ptolemaico en Cuadernos historia 16, 1985.

Flacelière, R.-ChAmbry. É.,

- Plutarque. Vies, texte établi et traduit par -, París, Tomes III (1964), IV (1966), V (1969), VI (1971), VII (1972), VIII (1973), XI (1976), XIII (1977), XV, (1979).

Frazier, F.-Froidefond, Ch.,

- Plutarque, Oeuvres Morales, La fortune des romains-La fortune d'Alexandre-La gloire des Athéniens, Tome V, Paris, 1990.

Geiger, J.,

- "Plutarch's Parallel Lives: The choice of Heroes", Hermes, 109 (1981) 85-104.

GuILLÉN, J.,

- Cicerón. Sobre los deberes, Madrid, 2001.

Helmbold, W. C.-O’Neil E. N.,

- Plutarch's Quotations, The American Philological Association, 1959.

HoldEN, H. A.,

- M. Tulli Ciceronis de Officiis libri tres, Amsterdam, 1966.
Hornblower, S.- Spawforth A. (eds.),

- The Oxford Classical Dictionary, Oxford University Press, $1996^{3}$.

López SAlvÁ, M.,

- Plutarco, Obras morales y de costumbres, V, Madrid, 1989.

LozAnO, A.,

- Las monarquías helenísticas I: El Egipto de los Lágidas, Madrid, 1989.

Manfredini, M.-Orsi, D. P.,

- Plutarco, Le Vite di Arato e di Artaserse, Fondazione Lorenzo Valla, 1996.

MiLler, W.,

- Cicero. De officiis, reimpr. de la ed. de 1913, Loeb, Londres-Cambridge Mass., 1997.

PERrin, B.,

- Plutarch's Lives, IV, reimpr. de la ed. de 1916, Cambridge Mass., 1968.

- Plutarch's Lives, V, reimpr. de la ed. de 1917, Cambridge Mass., 1961.

- Plutarch's Lives, VII, reimpr. de la ed. de 1919, Cambridge Mass., 1967.

- Plutarch's Lives, XI, reimpr. de la ed. de 1926, Cambridge Mass., 1975.

PORTER, W. H.,

- Plutarch's Live of Aratus, New York, 1979.

SimON, E.,

- Plutarque. Vies. Index des noms propres, tome XVI, Paris, 1983.

Stanwick, P. E.,

- Portraits of the Ptolemies, Greek Kings as Egyptian Pharaohs, University of Texas Press, 2003.

VV.AA.,

- Der kleine Pauly, 5 vols, Stuttgart y München, 1964-1975.

- Gran Enciclopedia Larousse, 1991.

WALKBANK, F. W.,

- A Historical Commentary on Polybius, vol. III, Oxford, 1979. 